\title{
Observations on Alveolar Pyorrhea and its Therapeutics in the Clinical Light
}

\author{
by
}

\author{
Masahiko TANIGUCHI*
}

From the clinical point of view, it may be stated that alveolar pyorrhea cases are to be classified as those of topical and those of general characters. The term 'alveolar pyorrhea' is the designation applied to the cases of topical character but it is also extended to cover those of general nature. However, one is essentially different from the other. It is true indeed that both topical and general alveolar pyorrhea cases give similar clinical findings in the parodontal tissues but the pathogenic causes for them are quite different from one another. Alveolar pyorrhea cases of general character occupy over $80 \%$ of occurrences and a curative effect becomes apparent only when remedy is administered for a topical alveolar pyorrhea in conjunction with a general one, whereas topical alveolar pyorrhea has nothing to do with general alveolar pyorrhea and as removal of the topical causes is sufficient to bring about a cure, it may be regarded as a kind of gingivitis due to mechanical stimulus.

Of various causes responsible for alveolar pyorrhea of topical character, mention first of all is made of dental calculi. It has been accepted as an established fact of long standing that dental calculi are responsible for the so-called alveolar pyorrhea. However, as a result of researches conducted by the author for years, it seems that these calculi have no direct influence on the incidence of alveolar pyorrhea but are only secondarily related to it. A survey of 100 cases of alveolar pyorrhea has revealed the fact that 77 were found with dental calculi and 23 with none at all. Although the majority were thus found with dental calculi, it was the remaining 23 cases that were alveolar pyorrhea cases of severe degree. Of 100 patients with dental calculi, 31 were alveolar pyorrhea cases, 37 were gingivitis cases and 32 were found normal. Based on these facts above, it may be concluded that the presence of dental calculi in the oral cavity does not always cause alveolar pyorrhea cases. The reason why it has been held that dental calculi are directly responsible for the incidence of alveolar pyorrhea may be that dental calculi are found in the mouth of alveolar pyorrhea patients to a high degree. In explanation of the above fact that dental calculi are found among the alveolar pyorrhea patients to a high degree, Professor Jiro Kanno, Department of Biochemistry, Nihon University School of Dentistry, holds that alveolar pyorrhea patients are in a state where they are apt to be suffered from dental calculi. It may be a possibility that dental calculi in the mouth induce an inflammation of gingivae and thus promote the progress of alveolar pyorrhea. It has been found the curative state of alveolar pyorrhea remains the same whether dental calculi are removed or otherwise. Other causes are found among the inadequate gold crowns, gold inlays, clasps of artificial teeth, abnormal alignment of teeth and

* 谷口 雅彥: Dept. of Biochemistry, Nihon Univ. School of Dentistry (Director: Prof. Jiro $K A N N O)$ 
excessive load of weight, etc., but they are cured by the removal of topical causes accompanied with a proper general therapy.

What the author means here by the so-called alveolar pyorrhea is that it is a type of the complaint which is not cured no matter what kind of topical therapy is applied and affects not only the parodontal tissues topically but also those of an entire tooth, ultimately leading to the fall of a tooth with constant deterioration throughout the process. The author has been long engaged in researches on the above type of alveolar pyorrhea and gives in the present report some of his findings relative to its clinical therapeutics.

As is already referred to, topical alveolar pyorrhea is distinguished from general alveolar pyorrhea by the fact that the former can be cured through the removal of topical causes. On the other hand, the latter has causes in the patient generally and for this reason a topical cure is insufficient. It is only when a general cure is administered that the progress of a complaint is checked or effects of a cure become apparent. The author came to be of the opinion that alveolar pyorrhea might be caused by the irregularity of internal secretions and a complaint of general character, finding its expression in the oral cavity. Therefore, he has used epidihydrocholesterol, prepared by Prof. Jiro Kanno of Nihon University School of Dentistry, in the clinical treatment of alveolar pyorrhea with the dose of $30-40 \mathrm{mg}$ for injection purposes. Epidihydrocholesterol is a direct precursory substance of steroid hormone and it is thought to transfer itself into steroid hormone in vital body. A recent report by Prof. Konno reveals that epidihydrocholesterol is found in the brains, heart and other organs of animals to a considerable degree and it is a question yet to be solved whether epidihydrocholesterol directly acts on the organs or exerts its action after changing itself into some kind of hormone. But it is thought that epidihydrocholesterol acts in these two ways. That a direct application of epidihydrocholesterol in the form of ointment on the gingival region quickens the cure of alveolar pyorrhea, attests to a supposition that it possesses a direct action.

The following findings have been obtained as a result of the clinical application of epidihydrocholesterol to 100 cases of alveolar pyorrhea including their internal complaints :

\begin{tabular}{lcl} 
〔Names of Complaints $]$ & [No. of Cases $]$ & \multicolumn{1}{c}{ [State of Cure $]$} \\
Stiffness in the shoulders & 83 & Almost cured or near recovery \\
Neuralgia & 26 & 17 cured/ others near recovery \\
Climacteric changes & 13 & Completely recovered \\
Liver troubles & 12 & Near recovery \\
Infertility & 3 & 1 near recovery \\
Infiltration of the lungs & 8 & 5 near recovery \\
Pregnancy & 2 & Slight vomit feeling \\
Diabetes & 8 & 2 near recovery
\end{tabular}

As is known from the above tabulation, $90 \%$ of alveolar pyorrhea cases have some sort of internal complaints and the application of epidihydrocholesterol proved quite effective for stiffness in the shoulders and climacteric changes. These considerations enable us to infer that the internal complaints are induced by an unbalance of hormone in the body.

Another survey, which dealt with relationship between cases of alveolar pyorrhea and those of internal troubles, has given the following findings. 


\section{Complaints}

Diabetes

Climacteric changes

Neuralgia

Infiltration of the lungs

Liver troubles

Mental dementia

Alcoholic addicts
No. of Cases

12
15
25
45
15
223
45

$\begin{array}{cc}\text { Those with } & \text { Percentage } \\ \text { Alveolar Pyorrhea } & \text { Per } \\ 12 & 100 \% \\ 12 & 80 \% \\ 9 & 35 \% \\ 21 & 45 \% \\ 12 & 80 \% \\ 136 & 60 \% \\ 35 & 75 \%\end{array}$

The above figures are evidence enough in favor of an inference that some relationship exists between alveolar pyorrhea and internal complaints. The complaints such as diabetes and climacteric changes which are believed to take place due to an insufficiency of hormone have shown high recovery rates, $100 \%$ and $80 \%$ respectively. It is considered that cases of liver troubles and alcoholism have close relation with alcohol and those of mental dementia $(60 \%)$ with partial proclivity of food consumption.

The following survey revealed relationship existing between age groups and alveolar pyorrhea patients.

$$
\begin{gathered}
\text { Age groups } \\
\text { Over } 40 \\
30-39 \\
20-29
\end{gathered}
$$$$
\text { Below } 20
$$

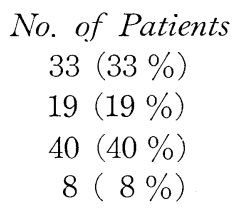

When figures for those over twenties and also those of twenty years of age are combined, they give a figure as high as $48 \%$. In dealing with the clinical treatment of alveolar pyorrhea, we should concentrate our efforts on the patients falling under these age brackets. It is a matter which claims our attention that nearly $70 \%$ of the cases of the so-called adolescent alveolar pyorrhea which occurs in a period when young people move from childhood into adolescence is attributed to their partial proclivity in food consumption. It is to be supposed that in this pericd the young people stand in need of far more hormone for their growth but they do not take in sufficient hormone because of their partial proclivity in food consumption, thus bringing about an unbalance in their hormone requirements. Among foods which they do not like are reported fishes, especially those rich in fat such as mackerels and sardines, and meat. Occurrences of internal troubles and those of alveolar pyorrhea are coincident in this period of adolescence and, for this reason, it is imperatively necessary that actions be taken to correct their partial likes in the matter of focd consumption both from treatment and prevention of internal troubles and alveolar pyorrhea as well. In the above table, those in the age bracket of 30 to 39 gave an incidence rate of 19 cases but this figure does not seem to represent a true picture, for in our works at health centers in contact with mothers we find a high incidence rate of about $80 \%$ in this age group. A reason for this high incidence figure among the pregnant mothers may be that much hormone is consumed in the growth of fetus, thereby causing deficiency in their hormone balance. Lastly, as regards those over 40 years of age an incidence of the so-called climacteric alveolar pyorrhea is very frequent and it is not hard to imagine that the gradual deterioration of various vital organs can not keep up with supply of the required hormone, its deficiency leading to susceptibility to diseases. What is interesting in this 
matter is that the climacteric period corresponds to what are traditionally known as dangerous years among the Japanese. To put it another way, it falls on a period when a deficiency of hormone usually starts.

The facts cited above lend support to the belief of the author that alveolar pyorrhea is an expression of complaints of general character. It follows then that a remedy of alveolar pyorrhea is not effective unless a general therapy is administered.

As is already mentioned, an application of epidihydrocholesterol checks the progress of alveolar pyorrhea and promotes the proliferaton of alveolar bones. This fact clearly attests to a relationship as exists between alveolar pyorrhea and hormone and the author should like to recommend the use of hormone therapy for the treatment of alveolar pyorrhea. The effectiveness of hormone is also true of internal complaints and it is thought advisable to take into full account balance picture of hormone in the initial stage of an internal trouble. A deficiency of hormone may act unfavorably to reduce the resistant power of man against bacteria and thus give rise to a chance of disease infection.

With referense to the therapeutics of alveolar pyorrhea, different methods are applied according as whether a case is topical or general.

As regards a case of general nature, three actions are suggested as follows :

1) To correct partial likes in the matter of food.

2) To abstain from the consumption of alcoholic beverages.

3) To make good an insufficiency of hormone through use of epidihydrocholesterol. 'Presteronsol' which is available on the market is a water solution preparation of epidihydrocholesterol but in an actual trial conducted by the author, it did not prove much of benefit. Therefore, it is urged that an oil preparation of epidihydrocholesterol should be used.

Hitherto there has been a belief that alveolar pyorrhea is a disease impossible of cure but in the opinion of the present writer it is merely due to lack of understanding that alveolar pyorrhea is a disease of general nature. When a disease is of general nature, it is of no use how much topical therapeutics is resorted to. On the other hand, when a topical treatment is followed by an adequate general treatment alveolar pyorrhea will be cured with relative ease.

Irrespective of a case of alveolar pyorrhea whether of general or topical nature, what is the most important consists in the removal of diseased gingivae. Even with general therapeutics it is not easy to effect a complete cure of diseased gingivae and for this reason the author has been advocating the removal of diseased gingivae and then help to promote granulosis. In a case of slight deterioration, the use of curettage is sufficient and when a case is severe enough an excision by means of surgical knife is the usual practice. As for himself, the writer has been using the removal method by cautery with satisfactory result, which method is accompanied with the following advantages.

a. It is easy of handling.

b. It has the least risk of infection.

c. Minimal bleeding at the time of an operation.

d. It enables a rapid recovery.

This cautery method is found effective not only when a case is of slight deterioration, but also when a case is severe. It saves the length of time usually required in an operation of alveolar pyorrhea. 
In conjunction with cautery, the writer uses two kinds of stop fillers, one with a vertical point and one with a horizontal point. When sufficiently heated, the cauterizing instrument is inserted into the diseased gingivae and scales them off. In a slight case, the cauterizing instrument is so manipulated as to softly touch the surface of the diseased region, while in a case of severe degree it is so used as to come into contact with alveolar bones. The diseased part of gingivae is very flaccid to the touch while, on the other hand, the normal part is felt to be hard and does not easily admit an insertion of an instrument. Therefore, ease or difficulty in the insertion of an instrument into the gingivae serves as an indication as to how far the diseased condition has advanced. Subsequent to cautery, scaling of dental calculi is carried out and depending upon the case, some paste dressing is administered. $10 \%$ solution of silver nitrate may be employed with a view to alleviating possible post-operational pains. An application of epidihydrocholesterol in the form of ointment is found to help largely in reducing pains and promoting the proliferation of granules. For a week or ten days following an operation some pain is inevitable but in about two weeks granulosis sets in. However, if it is left without any follow-up there is a dangerous possibility that new granules may change into pathogenic ones. As a preventive measure, the use of tooth brush proves beneficial in giving the granules proper amount of stimulus. A soft brush is recommended for the first two weeks to be followed by a hard brush afterward. If there should happen sporadic change of new granules into pathogenic ones, an application of epidihydrocholesterol paste will do a wonderful job in remedying them. It may be added that with regard to the upper jaw the entire teeth are operated in one sitting and those of the lower jaw in two sittings. As compared with an excision with surgical knife, the cautery method gives less injury to the normal part around the diseased region and this is histologically desirable.

A few comments are made on the Neumann method that is regarded as the only complete operation of alveolar pyorrhea cases. This surgical method is predicated on a belief that the cause of alveolar pyorrhea is sought in the parodontal tissues and the writer finds it very complicated that this method requires not only an excision of diseased gingivae but also an excision and smoothing out of alveolar bones. And in spite of complexities involved in this method the result of this operation is not always successful with a possible relapse. With the cautery method it is not necessary to seek as deep as alveolar bones and in many cases successful results have been reported where no alveolar bones were touched.

At any rate, either the Neumann method or the Black method is a treatment of topical nature neglectful of a general therapeutics. Once again need is stressed for a general therapy in the treatment of alveolar pyorrhea.

While a case of alveolar pyorrhea is still in the initial stage, there is no problem of extraction. But in proportion to the degree of the diseased conditions the problem of extraction comes to crop up. According to the present practice, when the root of a tooth still remains in the socket more than one half it is retained. Any case below this level is considered to call for an extraction. But this practice is based on a belief that alveolar pyorrhea is impossible of cure but when an assumption is made that alveolar pyorrhea is capable of being completely cured, this will result in a new theory. For this reason, what the writer propounds is to be understood as predicated on an assumption that cases of alveolar pyorrhea are possible of cure. From this point of view, an indication of extraction should be determined by some fixed apparatus irrespec- 
tive of a relation between root and alveolar bone as is the present practice. The criterion should be whether a given tooth is capable of being retained or not and, therefore, when it is possible, even one-fifth should be retained. For the writer is of the opinion that with an adequate treatment alveolar pyorrhea can be checked in its progress or cured with every possibility of alveolar bones proliferating and gradual rehabilitation of the masticatory function. Rehabilitation of the masticatory function is absolutely necessary, because no matter how well artificial teeth are prepared none excel the natural teeth.

Importance of a fixed apparatus in the determination of an extraction needs no further emphasis. When the deterioration is so advanced that a tooth moves itself, an apparatus has to be employed in an effort to fix it. As the author advocates nonextraction as far as possible, need of a fixed apparatus is keenly apparent. Advantages of this kind of apparatus are summarized as follows.

(1) Rehabilitation of the masticatory function.

(2) Ease of a surgical operation.

(3) Minimal difficult after an operation and rapid recovery.

(4) A strong possibility of proliferation of alveolar bones.

(5) Minimal danger of relapse.

Of course, different stages of deterioration call for different methods and scope of fixation. When the deterioration is much advanced, it is not seldom that the whole of fourteen teeth has to be fixed altogether.

The writer effects an extraction of pulp for the sake of ease in adjustment. When the primary objective in the treatment of alveolar pyorrhea is placed on the rehabilitation of the masticatory function, there is not involved much harm in the extraction of pulp. Anterior teeth are fixed with inlays while molar teeth are fixed with onlays on the occlusal surface. Recourse is also had to Carmichael attachment in the molar region but this should be decided according to the degree of deterioration. It is at the discretion of an operator to decide on a method which he considered safe and appropriate to a case on hand. In employing an attachment apparatus, care should be exercised not to give undue pressure on the teeth involved. Fixation of 6 anterior teeth or 4 molar teeth does not present much of a problem but it is not seldom when 10 or 14 teeth have to be fixed. What is important in fixation is that every inlay and onlay is made securely and accurately. Use of wax patterns is suggested when fixing 2 or 3 teeth at once but a result is not always favorable. In fixing 3 or 4 teeth, the usual practice is to take partial impressions by means of plaster. When partial impressions thus collected are put together, a result will come near to a perfection in fixation. It should be refrained from taking an impression extending over many teeth at one time. The reason for this is sought in the fact that changes take place on account of shrinkage of plaster and imperfection of heat-resistant agent for fixing purposes. When proper adjustment is thus made, a fixation of many teeth will not prove a difficult task. Usual materials are $18 \mathrm{~K}$ gold alloy and gold palladium, fixing being effected by means of $16 \mathrm{~K}$ gold wax. In the experience of the writer, gold alloys have been found most satisfactory.

\section{Conclusive Remarks}

In dealing with the clinical therapeutics of alveolar pyorrhea, we find it necessary 
to consider it in terms of the periods of incidence.

(The first incidence) Period from childhood to adolescence.

(The second incidence) Pregnancy of women.

(The third incidence) Period from the middle of life to old age.

It is a truth of statistical validity that when people are past the first and second periods the incidence of alveolar pyorrhea becomes less frequent. When completely cured, a case of alveolar pyorrhea does not suffer a relapse except in those who are constitutionally weak, alcoholic addicts or those partial in their food consumption. It seems that those who suffer from alveolar pyorrhea in the third stage of incidence in the above classification will encounter a relapse more frequently. It goes without saying that a relapse is influenced to a large degree by care in the daily life of the patient himself. Generally speaking, the post-operational results have been quite satisfactory. Treatment of alveolar pyorrhea should be looked at from a point of general therapeutic view and not confined to a topical application.

When a case of alveolar pyorrhea is of slight degree, removal of the diseased. gingivae and general therapeutics have been found quite sufficient. For this purpose, the use of epidihydrocholesterol in the form of oil injection is believed to be the ideal therapeutic means to bring about normalcy in the unbalance picture of hormone in the vital body. At the same time, the patient of alveolar pyorrhea should be encouraged to correct his partial proclivity in the matter of his daily focd consumption.

The degree of deterioration is to be taken into due consideration when an application of an attachment apparatus in called for but the same therapeutics is continued. However, when the use of an attachment apparatus means an economical burden on the part of a patient an extraction of the diseased granules is to be recommended while alveolar pyorrhea is in the initial stage of incidence. Among those who had suffered from a relapse after their treatment of alveolar pyorrhea are mentioned those who art intemperately addicted to alcohol, those who are partial in their food consumption, those who were contacted with some internal complaint of severe nature, those who became pregnant and gave a birth and so forth.

It has been found that a periodical injection of epidihydrocholesterol, twice or three times on a monthly basis, is much efficacy in checking possible relapse to a minimal degree. 